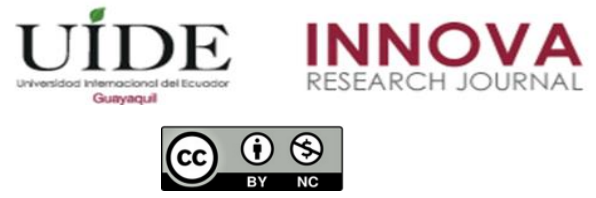

INNOVA Research Journal, ISSN 2477-9024

(Septiembre-Diciembre 2020). Vol. 5, No.3 pp. 1-21

DOI: https://doi.org/10.33890/innova.v5.n3.2020.1288

URL: http://revistas.uide.edu.ec/index.php/innova/index

Correo: innova@uide.edu.ec

\title{
Tipos de Innovación como Estrategias de Adaptación al Dinamismo de los Mercados
}

\section{Types of Innovation as Adaptation Strategies to the Dynamism of Markets}

Valeria Michelle Acosta Castillo

https://orcid.org/0000-0002-2777-1249

Bryan Andrés Vega Morejón

https://orcid.org/0000-0003-3415-8386

Mayiya Lisbeth González Illescas

http://orcid.org/0000-0002-5219-3807

Luis Pastor Carmenate Fuentes

http://orcid.org/0000-0001-6057-2746

Universidad Técnica de Machala, Ecuador

Autor para correspondencia: vacosta_est@utmachala.edu.ec; bavega_est@utmachala.edu.ec; mlgonzalez@utmachala.edu.ec; lcarmenate@utmachala.edu.ec

Fecha de recepción: 26 de diciembre del 2019 - Fecha de aceptación: 22 de abril del 2020

\section{Resumen}

La globalización y el dinamismo de los mercados presionan a las empresas a implementar diversas estrategias para defender su posicionamiento. La innovación sobresale como respuesta estratégica de las empresas para adaptarse a los cambios. Este trabajo se guía por el objetivo de identificar los tipos de innovación que las empresas están utilizando como estrategias de adaptación al dinamismo de los mercados. Se emplea un enfoque cualitativo mediante la aplicación de los métodos teóricos histórico-lógico y analítico-sintético para la interpretación de la literatura relevante. Se utilizan como fuentes de información 20 publicaciones científicas que cubren estudios de casos de empresas exportadoras que han aplicado prácticas organizacionales, a partir de las cuales se asocia a un tipo de innovación. Para el caso de Ecuador el análisis se centra en tres sectores productivos, tomando como referencia la representatividad de los productos de exportación. Entre los principales hallazgos se destaca que las empresas aplican la innovación abierta asociada a los procesos de certificación que requieren referentes externos de buenas prácticas, cooperación y transferencia de información. Finalmente se concluye que las empresas emplean mayoritariamente la innovación de tipo incremental, segueida de la innovación abierta y la innovación radical para responder a los cambios del entorno, mejorar el aprendizaje y aprovechar las oportunidades de los mercados globales.

Palabras claves: estrategia; competitividad; innovación; tipos de innovación; empresas exportadoras 


\begin{abstract}
Globalization and the dynamism of markets pressure companies to implement different strategies to defend their position. Innovation stands out as a strategic response from companies to adapt to change. This work is guided by the objective of identifying the types of innovation that companies are using as adaptation strategies to the dynamism of the markets. A qualitative approach is used by applying theoretical historical-logical and analytical-synthetic methods for the interpretation of the relevant literature. Twenty scientific publications are used as sources of information covering case studies of exporting companies that have applied organizational practices, from which a type of innovation is associated. In the case of Ecuador, the analysis focuses on three productive sectors, taking as a reference the representativeness of export products. Among the main findings is that companies apply open innovation associated with certification processes that require external reference of good practices, cooperation and information transfer. Finally, it is concluded that the majority of firms employ incremental innovation, followed by open innovation and radical innovation to respond to changes in the environment, improve learning and take advantage of global market opportunities.
\end{abstract}

Key words: strategy; competitiveness; innovation; types of innovation; export companies

\title{
Introducción
}

En el entorno comercial se manifiestan transformaciones asociadas en diferentes campos, situación que afecta el desempeño de las empresas que actúan en mercados domésticos e internacionales. Este escenario marca un desafío permanente a las empresas exportadoras, las cuales deben reorientar su gestión y decisiones estratégicas para ajustarse de forma pertinente a los cambios.

Ante la problemática esbozada, la innovación se destaca como una respuesta que permite a las empresas proyectar características diferenciadoras, las mismas que favorecen la generación de ventajas competitivas de mayor duración en el tiempo. En este sentido, la competitividad de las empresas exportadoras se asocia a la capacidad que estas tengan para innovar.

El tratamiento de la innovación como factor clave para el crecimiento económico no es nuevo. Así, a principios del siglo XX, Schumpeter destacaba en su análisis el papel protagónico de la innovación como un motor interno fundamental para el crecimiento económico de las empresas y naciones. Independientemente del origen de las empresas, la innovación de procesos, productos y servicios se constituye en una estrategia que permite proyectar mayor valor agregado a las empresas, aumentando las posibilidades de que puedan convertirse en organizaciones competitivas y logren adaptarse a las exigencias de los mercados mundiales.

Para el caso de las empresas ecuatorianas, la oferta exportable mayoritariamente de productos primarios se enfrenta a competidores fortalecidos por sus estrategias de diferenciación y la incorporación de nuevos competidores. En este sentido, las empresas que proyectan un buen desempeño en los mercados internacionales son un referente para el análisis de sus prácticas organizacionales relacionadas a la innovación.

En función de lo expuesto, nos planteamos el objetivo de identificar los tipos de innovación que las empresas están utilizando como estrategias de adaptación al dinamismo de 
los mercados. Para ello se realiza una revisión de artículos científicos, en donde se analiza la innovación en organizaciones de distintos países del mundo. La metodología empleada comprende un enfoque cualitativo descriptivo, bajo los métodos teóricos; histórico-lógico y analítico-sintético, y métodos empíricos; análisis de documentos clásicos. En la primera fase de resultados, se exponen los estudios realizados a las empresas de Latinoamérica y el resto del mundo, el contexto innovador y su enfoque según su impacto. En la segunda fase se analiza la innovación en empresas ecuatorianas.

La utilidad del presente trabajo se basa en proporcionar una mirada a las distintas perspectivas de la innovación y estrategias utilizadas por las organizaciones para mejorar su competitividad en los mercados dinámicos. El aporte al conocimiento de estas acciones podría conducir a los empresarios a mejorar la toma de decisiones y plantear en sus organizaciones estrategias idóneas para convertirse en empresas altamente competitivas.

La estructura del artículo comprende la introducción seguida de la revisión de literatura, en donde se destaca la perspectiva de varios autores tanto clásicos como contemporáneos sobre la innovación y su tipología. En el apartado de metodología se describe el enfoque utilizado, luego los resultados donde se exponen los principales hallazgos. Finalmente, en la sección de conclusiones se presenta algunas reflexiones en función de los resultados y tipo de investigación realizada, lo cual sirve de premisa para plantear futuras líneas de investigación.

\section{Revisión de la Literatura}

\section{La estrategia empresarial y la innovación}

Los cambios que se producen constantemente en el entorno de negocios, aumentan la complejidad de gestión de las empresas para defender su espacio en el mercado. Por ello, para la toma de decisiones se requiere considerar estrategias alternativas que contribuyan a la adaptación de las nuevas condiciones y a la generación de ventajas competitivas.

De acuerdo a la visión de autores clásicos como Chandler (1962) y Adrews (1971), el diseño de estrategias implica un análisis de la propia estructura organizacional y del entorno para definir planes acorde a las transformaciones del contexto. Esto es congruente con la premisa de March y Simon (1958), referida a que las decisiones están influenciadas por la información disponible. Ohmae (1989) define la estrategia como un conjunto de acciones planificadas para establecer factores diferenciadores de la competencia, considerando las necesidades de los clientes y del entorno en el que se desarrolla.

Así también, Porter (1980, 1985) a través de sus obras "Competitive Strategy" y "Competitive Advantage", influenciado bajo los principios de la Economía Industrial, priorizó el análisis de las fuerzas competitivas y del entorno para el diseño de estrategias genéricas como liderazgo en costos, diferenciación y enfoque. Cabe destacar que Porter (1990) reconociendo el dinamismo de los mercados, actualizó su enfoque de ventaja competitiva hacia una teoría dinámica de la estrategia en la obra "The Competitive Advantage of Nations". 
Bajo el objeto de nuestro estudio, entre las contribuciones de Porter (1980, 1985, 1990) destacamos su visión sobre la innovación como estrategia para aumentar la productividad de los recursos y alcanzar la competitividad.

En ese sentido, la innovación se interpreta como una estrategia de diferenciación, que se basa en crear o mejorar productos o servicios, adaptar procesos, implementar cambios en la gestión, lo cual aumenta las posibilidades de las empresas para obtener mejores resultados que la competencia (Chirinos y Rosado, 2016). Al implementar la innovación como estrategia de diferenciación, las empresas aspiran a que los clientes distingan las particularidades de sus productos y servicios y estén dispuestos a pagar más.

Al hilo de lo expuesto, ante el dinamismo de los mercados internacionales que se refleja en cambios acelerados de las fuerzas de la oferta y la demanda, las empresas exportadoras están obligadas a desarrollar habilidades que les permita identificar, planear y ejecutar propuestas innovadoras para el aprovechamiento de las oportunidades del mercado. (Montoya, 2004; Polo, Ramos, Arrieta y Ramirez, 2018).

\section{La innovación desde el enfoque empresarial}

Una extensa literatura asocia el término innovación a los resultados empresariales. Así, entre los estudios de mayor difusión y aceptación se destaca la obra de Schumpeter (1934) titulada "The Theory of Economic Development", en la cual relacionaba el nivel de producción con la combinación de distintos factores. Así mismo, atribuía a la innovación la introducción de novedades, como factores diferenciadores entre los competidores, lo que hacía posible sostener la diferencia de tasas de crecimiento entre las empresas.

Damanpour (1991) sostiene que la innovación abarca la creación de nuevos productos, extendiéndose a la incorporación de actualizaciones tecnológicas en procesos y en el ámbito de la gestión administrativa.

En el marco de la Teoría de la Empresa Basada en el Conocimiento, Nonaka y Takeuchi (1995) sostienen que la innovación es el proceso de negocio más intensivo en conocimiento, debido a que las empresas requieren renovar sus recursos disponibles y a su vez explorar el entorno para aprovechar el saber externo. Reconociendo que la prioridad de la gestión del conocimiento es mayor para las empresas exportadoras que para aquellas que actúan en los mercados domésticos (Gupta y Govindarajan, 2000).

La innovación en mercados es destacada en los estudios de Wang y Ahmed (2004) como un complemento necesario para direccionar el rendimiento de las empresas. Así, para mantener la competitividad en entornos de continuo cambio, al mejoramiento de productos y procesos se deben sumar novedosos enfoques para la introducción y explotación de nuevos mercados y nuevos clientes.

Con estas premisas y a manera de sistematización, en la tabla 1 vamos a exponer las contribuciones de varios autores clásicos y contemporáneos en una aproximación teórica a la innovación. 
Tabla 1.

La innovación desde un enfoque contemporáneo.

\begin{tabular}{|c|c|}
\hline Autores & Propuesta \\
\hline Schumpeter (1934) & $\begin{array}{l}\text { Lo define como una nueva forma de producción, abarca la creación de } \\
\text { un nuevo bien o proceso, la gestión de la organización, su estructura, } \\
\text { apertura de nuevos mercados, entre otros. }\end{array}$ \\
\hline Schmookler (1966) & $\begin{array}{l}\text { Surge tras la necesidad de solucionar un problema, las ideas creativas e } \\
\text { innovadoras responden a una demanda originada en el entorno. }\end{array}$ \\
\hline Sherman (1981) & $\begin{array}{l}\text { Ideas originadas tras identificar una necesidad, dando como resultado la } \\
\text { invención de nuevos productos, procesos o técnicas para lograr éxito en } \\
\text { el mercado. }\end{array}$ \\
\hline Nelson y Winter (1982) & $\begin{array}{l}\text { Consiste en implementar nuevos diseños a los productos, procesos o } \\
\text { procedimientos ya establecidos en las organizaciones con fines de crear } \\
\text { mejoras enfatizando en la incertidumbre como un riesgo por el cambio. }\end{array}$ \\
\hline Kanter (1984) & $\begin{array}{l}\text { La innovación contempla el cambio organizacional y los procesos de } \\
\text { aprendizaje para impulsar los cambios y actualizaciones. }\end{array}$ \\
\hline $\begin{array}{l}\text { Cardinal, Alessandri y Turner } \\
\text { (2001) }\end{array}$ & $\begin{array}{l}\text { Visualiza la innovación como un proceso que involucra aspectos } \\
\text { técnicos, físicos y actividades centrales basadas en el conocimiento, los } \\
\text { mismos que permiten crear rutinas organizacionales para el desarrollo de } \\
\text { productos. }\end{array}$ \\
\hline Herkema (2003) & $\begin{array}{l}\text { La innovación es esencialmente un proceso de conocimiento orientado a } \\
\text { crear nuevas ideas para el desarrollo de soluciones comerciales. }\end{array}$ \\
\hline Arraut (2008) & $\begin{array}{l}\text { Transformación de la estructura interna para la mejora del control y } \\
\text { gestión organizacional, implementación de herramientas tecnológicas } \\
\text { para la optimización de procesos y reducción de costos. }\end{array}$ \\
\hline Cano y Sánchez (2014) & $\begin{array}{l}\text { Uso de las capacidades y habilidades del recurso humano, y tecnología } \\
\text { en la cadena de valor con el fin de mejorar el proceso de producción. }\end{array}$ \\
\hline OECD y Eurostat $(2006,2018)$ & $\begin{array}{l}\text { Es la creación o mejoramiento de un producto, proceso o a su vez la } \\
\text { combinación de ambos. Así como también el uso de modernas técnicas } \\
\text { para la gestión y la comercialización. }\end{array}$ \\
\hline
\end{tabular}

Fuente: Elaboración propia.

Ante lo expuesto podemos distinguir que existen varias maneras de abordar la definición de la innovación. A partir de las referencias de la tabla 1, adoptamos la innovación como un proceso asociado al uso de las capacidades de las organizaciones para la creación de estrategias encaminadas a generar cambios que permitan crear o mejorar productos o servicios.

Así mismo, destacamos la gestión del conocimiento para alcanzar los objetivos de innovación, en paralelo a la transformación de la estructura organizacional que favorece la adopción de actualizaciones tecnológicas para la mejora de procesos de producción, la optimización de recursos, y la reducción de costos, con la finalidad de alcanzar el éxito económico en las empresas. 


\section{Tipos de innovación}

Si bien es cierto que el tratamiento de la innovación se remonta del siglo pasado, no existe un planteamiento preciso acerca de los tipos de innovación. No obstante, para los propósitos de este estudio, nos basamos en la clasificación de la innovación según su aplicación, planteada en el Manual de Oslo (OECD y Eurostat, 2006; 2018), según su impacto (Jordán, 2011) y según su paradigma (Chesbrough H. W., 2003). En la figura 1 se expone la clasificación descrita.

\section{Figura 1.}

Tipos de innovación desde el enfoque empresarial.

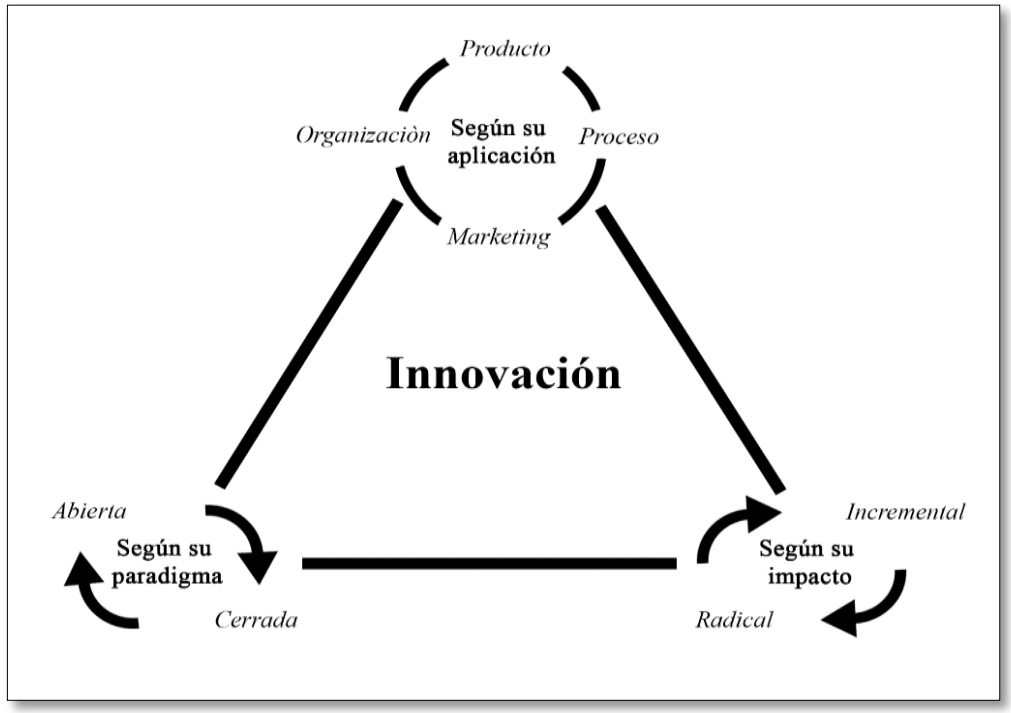

Elaboración propia a partir de (OECD y Eurostat, 2006, 2018; Jordán, 2011; Chesbrough H. W., 2003).

En el Manual de Oslo (OECD y Eurostat, 2006; 2018) se explica que la innovación según su aplicación se enfoca al mejoramiento o creación de nuevos productos; mejora de procesos de producción para la eficiencia de costos; mejora de procesos y eficiencia en ámbito comercial y mercadeo basado en preferencias de clientes; procesos de gestión y prácticas organizacionales, relaciones con empresas externas como soporte estratégico para innovar.

Por otro lado, según el grado de impacto, se identifica la innovación de tipo incremental y radical. Tras la relevancia para nuestro estudio, hemos ampliado la revisión bibliográfica de estos términos. En la tabla 2 exponemos las perspectivas de varios autores entre clásicos y contemporáneos acerca del tipo de innovación según su impacto.

Tabla 2.

Tipos de innovación según su impacto.

\begin{tabular}{ll}
\hline \multicolumn{1}{c}{ Autor/año } & \multicolumn{1}{c}{ Propuesta } \\
\hline & Ajustes y mejoras simples o menores en la tecnología en uso. \\
\cline { 2 - 2 } & $\begin{array}{l}\text { Es un perfeccionamiento progresivo tanto en productos como en } \\
\text { procesos, haciendo uso de recursos tecnológicos y de conocimientos ya } \\
\text { existentes. }\end{array}$ \\
\hline
\end{tabular}




\begin{tabular}{|c|c|c|}
\hline & Autor/año & Propuesta \\
\hline & Sen y Ghandforoush (2011) & $\begin{array}{l}\text { Se califica de adaptativa, su finalidad es la mejora de los procesos y } \\
\text { funcionalidades existentes. }\end{array}$ \\
\hline & Norman y Verganti (2014) & $\begin{array}{l}\text { Es hacer mejor lo que ya hacemos, ya sea en procesos, productos o } \\
\text { servicios. }\end{array}$ \\
\hline & Forés y Camisón (2016) & $\begin{array}{l}\text { Consiste en ejecutar pequeños cambios parciales, progresivos, } \\
\text { mejorando productos o procesos ya existentes en el mercado. }\end{array}$ \\
\hline & Kang y Hwang (2019) & $\begin{array}{l}\text { Se enfoca en mejorar productos o servicios basado en las necesidades } \\
\text { actuales de los clientes. }\end{array}$ \\
\hline \multirow{6}{*}{ 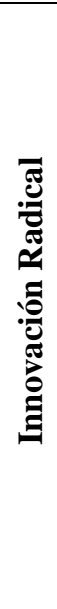 } & $\begin{array}{l}\text { Ettlie, Bridges y O'Keefe } \\
\text { (1984) }\end{array}$ & $\begin{array}{l}\text { Adopción de nueva tecnologia como estrategia de cambio estructural } \\
\text { para el desarrollo de procesos, formas de producción o servicios en las } \\
\text { organizaciones. }\end{array}$ \\
\hline & Dewar y Dutton (1986) & $\begin{array}{l}\text { Cambios drásticos, revolucionarios integrando conocimiento para el } \\
\text { desarrollo tecnológico. }\end{array}$ \\
\hline & Norman y Verganti (2014) & $\begin{array}{l}\text { Introducción de idea no aplicada anteriormente, tanto en procesos como } \\
\text { en productos. Las ideas innovadoras deben ser diferentes a las ya } \\
\text { existentes, contribuyen en la creación de iniciativas futuras. }\end{array}$ \\
\hline & Forés y Camisón (2016) & $\begin{array}{l}\text { Se enfoca en realizar productos o procesos completamente nuevos, } \\
\text { presentando diferencias relevantes en lo que corresponde a su propósito, } \\
\text { características y elementos utilizados para su elaboración. }\end{array}$ \\
\hline & Shih (2018) & $\begin{array}{l}\text { Consiste en efectuar un cambio completo a nuevos productos o servicios } \\
\text { que se introduciran en el mercado. }\end{array}$ \\
\hline & Kang y Hwang (2019) & $\begin{array}{l}\text { Modaliad de enfocarse en nuevos mercados desarrollando productos } \\
\text { nuevos y novedosos. }\end{array}$ \\
\hline
\end{tabular}

Fuente: Elaboración propia.

En las propuestas expuestas en la tabla 2 podemos observar que los autores coinciden. Por un lado, la innovación incremental, indiferentemente del contexto de aplicación (producto, proceso, organización) se enfoca en el perfeccionamiento paulatino y progresivo de estos factores a partir de funcionalidades existentes. López, Minguela, Rodríguez y Sandulli (2006) mencionan que su efectividad permite maximizar rentabilidad. Se caracteriza por no requerir alta inversión y por su bajo riesgo.

A diferencia, la innovación radical consiste en la introducción de nuevos productos, servicios o procesos, enfocados tanto en producción como en estructura organizacional, desde la implementación de una estrategia diferenciadora que le permita a la empresa competir a partir de una iniciativa novedosa. Se caracteriza por su significativo riesgo e inversión.

Bernal y Blanco (2017) acotan que la innovación incremental es imprescindible para decidir adoptar innovación radical desde una perspectiva que sea admisible para empresas que están tratando de crear productos no existentes en el mercado, pues si no existiese la innovación radical la innovación incremental alcanzaría el límite, y sin la existencia de esta, no se captaría el potencial dado por el cambio radical.

Finalmente, Chesbrough (2003) se enfoca en la innovación según su paradigma, el cual plantea que las organizaciones para alcanzar sus objetivos se basan en diferentes estrategias de innovación que conducen a la innovación cerrada y a la innovación abierta.

Inicialmente las empresas adoptaban innovación tradicional o cerrada, la cual consistía en estrategias verticales en donde las actividades se desarrollaban de manera exclusiva a nivel 
interno, es decir dentro de la organización (Chesbrough, 2003; Jamett, Alvarado y Maturana, 2017). En otro estudio Chesbrough y Crowther (2006) indican que la innovación cerrada es un modelo clásico en donde los proyectos de investigación, creación de ideas y desarrollo, se gestionan únicamente a partir del conocimiento y los recursos internos que posee la empresa, en donde la implementación de estos nuevos productos y procesos eran originarios de su organización.

Alvarez-Arós y Álvarez (2018) concuerdan con esta lógica y aportan que los elementos que caracterizan a la innovación cerrada son: uso del conocimiento solo a nivel interno, uso de fuentes verticales e investigación y desarrollo interno bajo riesgos propios, entre otros (Greco, Grimaldi y Cricelli, 2016).

Ante lo mencionado podemos decir que las estrategias para la innovación han presentado una evolución desde la innovación cerrada hacia la innovación abierta. Así, Chesbrough (2003) y Jamett et al., (2017) coinciden en señalar que el paradigma de la innovación abierta surge con la globalización, el dinamismo de los mercados y el fortalecimiento de I\&D, permitiéndoles a las organizaciones ampliar sus oportunidades.

En consecuencia, actualmente la tendencia está marcada hacia el uso de la innovación abierta como estrategia competitiva de crecimiento y desarrollo económico. En este contexto, la integración del conocimiento interno y externo permite aumentar las posibilidades de generar ventajas competitivas en las empresas (Badir, Frank y Bogers, 2019).

En la tabla 3 se recogen las apreciaciones de varios autores acerca de la innovación abierta que van desde lo clásico a lo contemporáneo.

Tabla 3.

Definiciones de Innovación abierta.

\begin{tabular}{ll}
\hline Autor/Año & Propuesta \\
\hline Teece (1986) & $\begin{array}{l}\text { Aprovechar el conocimiento tanto interno como externo y en cooperación con } \\
\text { agentes externos crear productos y procesos innovadores. }\end{array}$ \\
\hline Chesbrough (2003) & $\begin{array}{l}\text { Panorama de abundante uso del conocimiento, tanto interno como externo de las } \\
\text { empresas, con el fin de extraer el mayor valor de su potencial acelerando el proceso } \\
\text { de innovación. }\end{array}$ \\
\hline $\begin{array}{l}\text { Baldwin y Von Hippel } \\
\text { (2009) }\end{array}$ & $\begin{array}{l}\text { Proyectos colaborativos, los resultados producto de la investigación son de interés } \\
\text { colectivo, de libre acceso. }\end{array}$ \\
\hline $\begin{array}{l}\text { San Martín y } \\
\text { Rodríguez (2012) }\end{array}$ & $\begin{array}{l}\text { Antítesis de la innovación tradicional, creación de valor a partir del conocimiento } \\
\text { interno y externo. }\end{array}$ \\
\hline $\begin{array}{l}\text { Gómez y Etxebarria } \\
\text { (2012) }\end{array}$ & $\begin{array}{l}\text { Se lleva a cabo, a partir de reconocer que las organizaciones no consiguen trasformar } \\
\text { sus productos y servicios de forma aislada, por lo tanto, requieren permanentemente } \\
\text { obtener ideas, opiniones y recursos del entorno exterior de las empresas. }\end{array}$ \\
\hline $\begin{array}{l}\text { Álvarez y Bernal } \\
\text { (2017) }\end{array}$ & $\begin{array}{l}\text { Busca revelar la importancia y el modo como las empresas pueden ser capaces de } \\
\text { desarrollar su capacidad de innovación a través del aprovechamiento del } \\
\text { conocimiento interno y externo, para combinarlos y realizar proyectos de } \\
\text { investigación y desarrollo. }\end{array}$ \\
\hline A nivel empresarial, la innovación abierta destaca las dos principales e importantes \\
fuentes de conocimiento, internas y externas, que, al fusionarlas, permitirán innovar \\
y mejorar las posibilidades competitivas de la empresa.
\end{tabular}

Fuente: Elaboración propia. 
El análisis de los distintos planteamientos descritos en la tabla 3, permite sintetizar que la innovación abierta surge tras un concepto antagónico de la innovación cerrada, primando como factor clave el uso del conocimiento interno y externo o la combinación de ambos para la creación de valor, permitiendo acelerar los procesos de innovación y la competitividad ante los mercados dinámicos actuales.

Da Silva et al. (2016) señalan que la innovación abierta lleva a la organización a transformar el modelo de la competitividad y combinarlo con el modelo de la cooperación de todos los actores internos y externos en la búsqueda de la excelencia. Por otro lado Pavón, Hernández, Suárez, Jiménez y Sánchez (2015) mencionan que la innovación abierta mejora cuando se relacionan tres elementos clave; liderazgo basado en experiencia, uso de las Tics y agentes intermediarios.

Desde la perspectiva de las empresas Enkel, Gassmann y Chesbrough (2009) clasifican a la innovación abierta en tres procesos para el desarrollo: entrante (inside / inbound), saliente (outside / outbound) y mixtas (coupled), en la tabla 4 se exponen conceptos desde la perspectiva de varios autores.

Tabla 4.

Definiciones de los tipos de innovación abierta.

\begin{tabular}{|c|c|c|}
\hline & Autor/año & Aporte \\
\hline \multirow{3}{*}{ 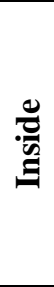 } & Chesbrough (2003) & $\begin{array}{l}\text { Se genera conocimiento dentro de los límites de una empresa para luego ese } \\
\text { conocimiento ser comercializado a otras empresas. }\end{array}$ \\
\hline & $\begin{array}{l}\text { Inauen y Schenker } \\
(2012)\end{array}$ & $\begin{array}{l}\text { Hace referencia a como la propiedad intelectual puede ser comercializable y } \\
\text { generar ganancias a través de la distribución de fuentes externas a la empresa. }\end{array}$ \\
\hline & $\begin{array}{l}\text { Alvarez y Álvarez } \\
(2018)\end{array}$ & $\begin{array}{l}\text { El recurso humano juega un papel fundamental en el manejo de ideas, } \\
\text { conocimiento científico y tecnología hacia adentro de los límites de una empresa, } \\
\text { se enfoca a conseguir recursos intangibles. }\end{array}$ \\
\hline \multirow{3}{*}{ 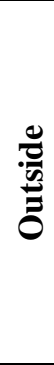 } & Chesbrough (2003) & $\begin{array}{l}\text { Aprovechar el conocimiento que ofrece el entorno externo, para } \\
\text { internalizarlo en la organización e integrarlo en su propio proceso de innovación. }\end{array}$ \\
\hline & $\begin{array}{l}\text { Inauen y Schenker } \\
(2012)\end{array}$ & $\begin{array}{l}\text { Estrategia que se basa en la búsqueda y adopción de nuevas ideas y tecnologías } \\
\text { de fuentes externas, que, con el fomento de actividades internas de investigación } \\
\text { y desarrollo, pueden generar ideas innovadoras. }\end{array}$ \\
\hline & $\begin{array}{l}\text { Alvarez y Álvarez } \\
(2018)\end{array}$ & $\begin{array}{l}\text { Se realiza por medio de la interacción con fuentes externas a la empresa como } \\
\text { son consumidores, proveedores, terciarios, institutos, centros de investigación, } \\
\text { mentores, subcontratación de Investigación y desarrollo, gobierno, se enfoca en } \\
\text { materializar los resultados de la innovación. }\end{array}$ \\
\hline \multirow{2}{*}{ 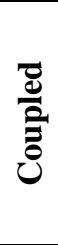 } & $\begin{array}{l}\text { Enkel, Gassmann, y } \\
\text { Chesbrough (2009) }\end{array}$ & $\begin{array}{l}\text { Combinación conjunta de actividades entrantes adoptando conocimiento externo } \\
\text { hacia adentro y salientes, direccionando hacia afuera las ideas propuestas e } \\
\text { implementarlas al mercado. }\end{array}$ \\
\hline & $\begin{array}{l}\text { Álvarez y Álvarez } \\
\text { (2018) }\end{array}$ & $\begin{array}{l}\text { Combinación simultanea de la innovación abierta de entrada y salida, enfocados } \\
\text { en la explotación conocimiento e innovación tecnológica en colaboración con } \\
\text { agentes externos. }\end{array}$ \\
\hline
\end{tabular}

Fuente: Elaboración propia.

Las organizaciones pueden plantearse alternativas encaminadas a fortalecer su competitividad adoptando estrategias a partir de la innovación abierta. Se identifican dos perspectivas diferenciales, en la primera denominada de dentro hacia fuera o saliente, prima 
como base el uso del conocimiento interno dentro de los límites de la empresa. Inauen y Schenker (2012) mencionan que las ideas e invenciones generadas a partir de estas estrategias pueden ser libremente comercializadas externamente, es decir fuera de la organización.

Por otro lado, la innovación de afuera hacia dentro o entrante, se orienta desde agentes externos, su enfoque es mucho más amplio y permite alianzas intersectoriales que pueden ser alianzas estratégicas con socios, proveedores e inclusive con la competencia. Su finalidad, innovar a partir del conocimiento de un capital humano experimentado y capacitado, fusionado con el uso de la tecnología de vanguardia y en caso de requerir, financiamiento para la investigación y desarrollo de la innovación.

Como una tercera práctica de innovación se identifica la innovación mixta o de cocreación, se refiere a la combinación de estrategias tanto de salida como, de entrada, esto permite la adquisición de conocimiento e innovación externa para procesarla internamente y lanzar al mercado ideas creativas con el fin de comercializarlas. Alvarez y Álvarez (2018) enfatizan en que no se puede aplicar innovación saliente sin antes adoptar innovación de entrada y que para aplicar estas estrategias se requiere inversiones significativas.

\section{Metodología}

A partir del objetivo planteado que consiste en identificar los tipos de innovación que las empresas están utilizando como estrategias de adaptación ante el dinamismo de los mercados, esta investigación se define de carácter descriptivo, con un enfoque cualitativo.

Se emplean los métodos teóricos como el histórico lógico y el analítico sintético para el análisis e interpretación de los planteamientos de los autores seleccionados en la revisión de literatura.

Se utilizan como fuentes de información las publicaciones científicas extraídas de prestigiosas bases de datos que integran autores clásicos y contemporáneos.

Los resultados están divididos en dos secciones. En la primera se realiza un análisis a diez publicaciones académicas que contemplan los casos de empresas latinoamericanas y del resto del mundo, pertenecientes a diversos sectores económicos. Se identifica el tipo de innovación aplicada por las empresas.

En la segunda sección de resultados se distingue el tipo de innovación que caracteriza a las empresas ecuatorianas. Tomando como base la revisión de literatura expuesta, se procede a identificar y clasificar el tipo de innovación, para ello nos enfocamos en las acciones implementadas desde tres sectores productivos. Cabe indicar que la fuente de información corresponde a publicaciones nacionales que han recogido la realidad de las empresas exportadoras. 


\section{Resultados y discusión}

En el contexto actual de mercados dinámicos, las empresas pugnan por defender su competitividad. De tal manera que buscan orientar sus prácticas organizacionales para atender los cambios, mejorar el aprendizaje de buenas prácticas de la competencia y aprovechar las oportunidades de los mercados globales.

Guiados por el propósito de esta investigación, en la tabla 5 se recogen diez estudios realizados por otros autores, los mismos que cubren casos de empresas exportadoras que aplicaron prácticas organizacionales de innovación. Con esta exposición y análisis pretendemos identificar los tipos de innovación que están empleando las empresas para robustecer sus ventajas competitivas.

Tabla 5.

Estudios de innovación en empresas de Latinoamérica y del resto del mundo.

\begin{tabular}{|c|c|c|c|}
\hline Autor/año & Muestra/Sector/País & Principales resultados & $\begin{array}{l}\text { Tipo de } \\
\text { innovación }\end{array}$ \\
\hline $\begin{array}{l}\text { Rodríguez, } \\
\text { Terán y Bucci } \\
\text { (2011) }\end{array}$ & $\begin{array}{l}30 \text { industrias Pymes, } \\
\text { sector metalmecánico, } \\
\text { Venezuela }\end{array}$ & $\begin{array}{l}\text { Identifica las prácticas de gestión para la innovación } \\
\text { abierta a partir de variables principalmente } \\
\text { investigación y desarrollo, la cual concluye que estas } \\
\text { empresas no cuentan con un departamento dedicado, } \\
\text { sin embargo, las empresas hacen todo su esfuerzo por } \\
\text { aprovechar oportunidades para innovar desde la } \\
\text { estructura organizacional con apoyo tanto interno } \\
\text { como externo. Se identifica la relación con otras } \\
\text { empresas, proveedores, universidades e institutos. }\end{array}$ & $\begin{array}{l}\text { Innovación } \\
\text { incremental; } \\
\text { abierta }\end{array}$ \\
\hline $\begin{array}{l}\text { Gálvez y } \\
\text { García (2012) }\end{array}$ & $\begin{array}{l}60 \text { Mipymes, sector de } \\
\text { software, ingeniería } \\
\text { eléctrica, artes gráficas } \\
\text { y plásticos, Colombia }\end{array}$ & $\begin{array}{l}\text { Analiza el rendimiento de la innovación en las } \\
\text { Mipymes, concluye que genera mayor rendimiento la } \\
\text { innovación en productos y procesos mientras que la } \\
\text { de gestión no genera un impacto significativo. }\end{array}$ & $\begin{array}{l}\text { Innovación } \\
\text { incremental, } \\
\text { radical }\end{array}$ \\
\hline $\begin{array}{l}\text { Inauen y } \\
\text { Schenker } \\
(\mathbf{2 0 1 2})\end{array}$ & $\begin{array}{l}141 \text { empresas de } \\
\text { tecnología, Alemania, } \\
\text { Suiza, Austria }\end{array}$ & $\begin{array}{l}\text { Analiza los paradigmas de la innovación sobre firmas } \\
\text { de tecnología. Las empresas que aplicaron } \\
\text { innovación cerrada presentaron mayor probabilidad } \\
\text { de producir innovación incremental. Por otro lado, } \\
\text { las que aplicaron innovación abierta con la estrategia } \\
\text { de código abierto mostraron mayor probabilidad para } \\
\text { el desarrollo de innovación radical, así mismo resalta } \\
\text { que entre más abierta es la innovación mayor es la } \\
\text { cantidad de innovación en procesos. }\end{array}$ & $\begin{array}{l}\text { Innovación } \\
\text { incremental, } \\
\text { radical; } \\
\text { abierta, } \\
\text { cerrada. }\end{array}$ \\
\hline $\begin{array}{l}\text { Tudor, } \\
\text { Zaharie y } \\
\text { Osoian (2014) }\end{array}$ & $\begin{array}{l}30 \text { pymes sector } \\
\text { fabricación, Cluj- } \\
\text { Rumania }\end{array}$ & $\begin{array}{l}\text { Aunque el estudio no determina el tipo de innovación } \\
\text { aplicado por las empresas estudiadas, se identifica el } \\
\text { uso de la innovación abierta en productos y procesos, } \\
\text { concluyendo que estas empresas usaron como } \\
\text { fuentes para generar ideas a partir de clientes y } \\
\text { proveedores en su mayoría; organización, } \\
\text { consultores externos y estado en término medio y } \\
\text { universidades e institutos en menor medida. }\end{array}$ & $\begin{array}{l}\text { Innovación } \\
\text { abierta }\end{array}$ \\
\hline $\begin{array}{l}\text { Bernal y Frost } \\
(2015)\end{array}$ & $\begin{array}{l}53 \text { empresas medianas } \\
\text { y grandes, sector } \\
\text { alimenticio, } \\
\text { manufactura, servicios, }\end{array}$ & $\begin{array}{l}\text { En este estudio, el } 67.4 \% \text { las empresas ejecutan dos } \\
\text { o más innovaciones en el año, de las cuales el } 4.3 \% \\
\text { innovaron para el mercado internacional; el } 82.6 \% \\
\text { realizaron cambios a las actividades de }\end{array}$ & $\begin{array}{l}\text { Innovación } \\
\text { incremental, } \\
\text { radical; } \\
\text { abierta }\end{array}$ \\
\hline
\end{tabular}




\begin{tabular}{|c|c|c|c|}
\hline Autor/año & Muestra/Sector/País & Principales resultados & $\begin{array}{l}\text { Tipo de } \\
\text { innovación }\end{array}$ \\
\hline & $\begin{array}{l}\text { petróleo y } \\
\text { construcción, } \\
\text { Colombia }\end{array}$ & $\begin{array}{l}\text { mercadotecnia y el } 27 \% \text { aplicaron mejoras en su } \\
\text { gestión empresarial. Así mismo, el } 27 \% \text { se } \\
\text { relacionaron con agentes externos para innovar; el } \\
77.1 \% \text { desarrollaron proyectos en departamentos de } \\
\text { investigación. Concluyen que las innovaciones } \\
\text { fueron más incrementales que radicales. }\end{array}$ & \\
\hline $\begin{array}{l}\text { Tarapuez, } \\
\text { Guzmán y } \\
\text { Parra (2016) }\end{array}$ & $\begin{array}{l}27 \text { empresas, sector } \\
\text { industrial, Colombia }\end{array}$ & $\begin{array}{l}\text { Las empresas analizadas cuentan con una estructura } \\
\text { flexible organizacional previamente definida, en } \\
\text { donde se aplicaron estrategias tanto reactivas como } \\
\text { proactivas, así mismo ciertas empresas se apoyaron } \\
\text { de agentes externos para el desarrollo interno, otras } \\
\text { por otro lado se basaron en la innovación } \\
\text { diversificando productos y servicios. }\end{array}$ & $\begin{array}{l}\text { Innovación } \\
\text { incremental; } \\
\text { abierta }\end{array}$ \\
\hline $\begin{array}{l}\text { García, } \\
\text { Gálvez y } \\
\text { Maldonado } \\
(2016)\end{array}$ & $\begin{array}{l}386 \text { empresas; Alianza } \\
\text { del Pacífico (Chile, } \\
\text { Colombia, México, y } \\
\text { Perú) }\end{array}$ & $\begin{array}{l}\text { Estudio enfocado en corroborar la incidencia de la } \\
\text { innovación en productos o servicios, procesos y } \\
\text { gestión, y su efecto sobre las ventas, empleo y } \\
\text { utilidades de las empresas. En donde se pone en } \\
\text { evidencia que Colombia es el país que más crea y } \\
\text { mejora productos, seguido de México con su } \\
\text { innovación en procesos y Chile con la innovación en } \\
\text { gestión Concluye además que la actividad } \\
\text { innovadora con recursos tanto internos como } \\
\text { externos repercute directamente en el crecimiento y } \\
\text { rendimiento de la misma, es decir su efecto es } \\
\text { positivo. }\end{array}$ & $\begin{array}{l}\text { Innovación } \\
\text { incremental, } \\
\text { radical; } \\
\text { abierta }\end{array}$ \\
\hline $\begin{array}{l}\text { Guisado et al., } \\
\text { (2016) }\end{array}$ & $\begin{array}{l}530 \text { empresas, sector } \\
\text { manufactura y } \\
\text { servicios, España }\end{array}$ & $\begin{array}{l}\text { El estudio se enfoca en el análisis sobre la } \\
\text { productividad laboral en donde se identifica que } 297 \\
\text { empresas son de baja productividad y } 233 \text { de alta } \\
\text { productividad realizan innovaciones incrementales y } \\
\text { radicales. En donde señalan que la innovación radical } \\
\text { afecta positiva y significativamente en comparación } \\
\text { con la incremental, así mismo añaden que la } \\
\text { innovación se relaciona con la productividad y que } \\
\text { para ello requiere de mano de obra cualificada. }\end{array}$ & $\begin{array}{l}\text { Innovación } \\
\text { incremental, } \\
\text { radical }\end{array}$ \\
\hline $\begin{array}{l}\text { Foronda } \\
(2018)\end{array}$ & $\begin{array}{l}431 \text { empresas sector } \\
\text { manufactura y } \\
\text { servicios, Bolivia }\end{array}$ & $\begin{array}{l}\text { El } 40 \% \text { de las empresas mejoró, implementó un bien } \\
\text { o servicio, por otro lado, el } 27 \% \text { realizó innovación } \\
\text { en procesos como resultado de inversión en bienes de } \\
\text { capital y maquinaria. }\end{array}$ & $\begin{array}{l}\text { Innovación } \\
\text { incremental y } \\
\text { radical }\end{array}$ \\
\hline $\begin{array}{l}\text { Rajapathiran } \\
\text { y Hui (2018) }\end{array}$ & $\begin{array}{l}379 \text { gerentes de } \\
\text { empresas de seguros, } \\
\text { Sri Lanka }\end{array}$ & $\begin{array}{l}\text { Concluyen que en el sector de seguros la capacidad } \\
\text { de innovación impacta positivamente en resultados } \\
\text { ya sea, si se aplica innovación mediante la creación o } \\
\text { mejora de productos, procesos, marketing u } \\
\text { organizacional. Enfatiza en la importancia de } \\
\text { desarrollar un comportamiento innovador dentro de } \\
\text { las organizaciones con los que puedan identificar } \\
\text { oportunidades del entorno y necesidades de los } \\
\text { clientes. }\end{array}$ & $\begin{array}{l}\text { Innovación } \\
\text { incremental } \\
\text { radical; } \\
\text { abierta }\end{array}$ \\
\hline
\end{tabular}

Fuente: Elaboración propia

En los trabajos que sirven de referencia para el análisis se destaca que las empresas latinoamericanas involucradas aplican mayoritariamente la innovación de tipo incremental, seguida de la innovación radical y la innovación abierta. 
De acuerdo a los casos analizados, la innovación incremental se caracteriza por su enfoque en productos o servicios, procesos y organización. Se revela que las empresas realizan una mejora y optimizan recursos en sus procesos productivos.

La innovación de tipo radical se vincula a la diversificación de nuevas líneas de productos o servicios, la implementación de tecnología que favorece la modificación de procesos de producción, formas de organización y en el acceso a nuevos mercados internacionales (Foronda, 2018; Bernal y Frost, 2015; García et al., 2016).

La innovación abierta se caracteriza con las variables aplicación del conocimiento, colaboración con agentes tanto internos como externos para la investigación y desarrollo empresarial, alianzas estratégicas con otras organizaciones en las que se incluyen proveedores, competencia, consultores, estado, universidad (Rodríguez et al., 2011; Bernal y Frost, 2015; Tarapuez et al., 2016; García et al., 2016). Por otro lado, Tudor et al. (2014) en su estudio a empresas de producción de Rumania identifican el grado de utilidad de fuentes para innovar, concluyendo que se basan en agentes externos, entre ellos, clientes y proveedores en mayor grado, seguido por consultores y entidades gubernamentales y en menor medida institutos y universidades.

De acuerdo a Lakhani, Lifshiz Assaf y Tushman (2013), la innovación abierta se aplica significativamente en el sector de software y tecnología, en donde la estrategia de código abierto brinda apertura a la cooperación del talento externo que contribuye con sus conocimientos al desarrollo de un proyecto común.

Por lo tanto, ante lo analizado evidenciamos que el sector de la tecnología a nivel mundial explota en mayor medida la innovación abierta desde un enfoque saliente (dentro hacia fuera), mientras que en otros sectores productivos aplica innovación de entrada o mixta para mejorar su competitividad.

Al hilo de los hallazgos relacionados a las investigaciones citadas, en una segunda fase de análisis de resultados nos enfocamos en las prácticas de las empresas exportadoras ecuatorianas, a partir de las cuales se asocian a un tipo de innovación. Para ello se examina a tres sectores productivos, tomando como referencia la representatividad de los productos de exportación.

Tabla 6.

Tipos de innovación en empresas exportadoras de Ecuador

Sector/ Producto Acciones implementadas

Tipo de innovación aplicada

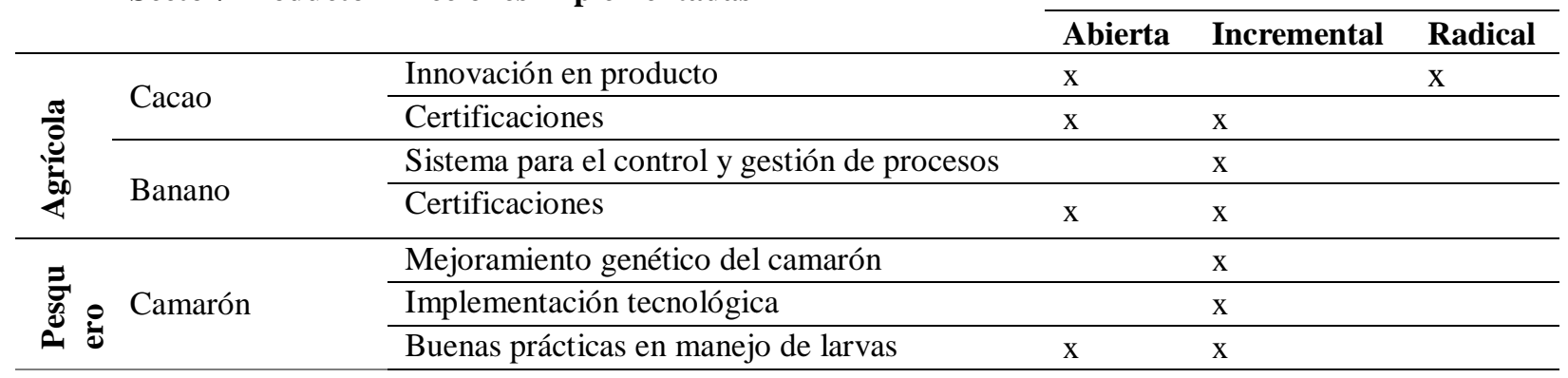

Esta obra se comparte bajo la licencia Creative Common Atribución-No Comercial 4.0 International (CC BY-NC 4.0) Revista de la Universidad Internacional del Ecuador. URL: https://www.uide.edu.ec/ 


\begin{tabular}{|c|c|c|c|c|c|}
\hline & \multirow{4}{*}{ Sector/ Producto } & \multirow{2}{*}{ Acciones implementadas } & \multicolumn{3}{|c|}{ Tipo de innovación aplicada } \\
\hline & & & Abierta & Incremental & Radical \\
\hline & & Mejora en la nutrición de larvas & & $\mathrm{x}$ & \\
\hline & & $\begin{array}{l}\text { Capital intelectual como plataforma para } \\
\text { innovar }\end{array}$ & $\mathrm{x}$ & & \\
\hline \multirow{9}{*}{ 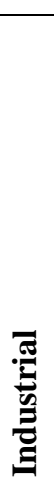 } & \multirow{3}{*}{ Textil } & $\begin{array}{l}\text { Implementación maquinaria de punta para } \\
\text { hiladas }\end{array}$ & & $\mathrm{x}$ & \\
\hline & & Implementación de tecnología & & $\mathrm{X}$ & \\
\hline & & Innovación en producto & & $\mathrm{x}$ & \\
\hline & \multirow{2}{*}{ Carrocería } & Implementación de nueva tecnología & & $\mathrm{x}$ & \\
\hline & & Eficiencia en los procesos productivos & & $\mathrm{x}$ & \\
\hline & \multirow{4}{*}{$\begin{array}{l}\text { Alimentos y } \\
\text { bebidas }\end{array}$} & Innovación en productos & & $\mathrm{x}$ & $\mathrm{X}$ \\
\hline & & Mejora en productos y procesos productivos & & $\mathrm{x}$ & \\
\hline & & Mejoras en estructura organizacional & & $\mathrm{x}$ & \\
\hline & & $\begin{array}{l}\text { Mejora en técnicas de promoción y fijación } \\
\text { precio }\end{array}$ & & $\mathrm{x}$ & \\
\hline
\end{tabular}

Fuente: Elaboración propia a partir de Ekos (2012; 2019), Paspuel (2015), Escobar (2016), Líderes (2017), Bonilla, Oña y López (2018), Vistazo (2018), Revista Gestión (2018), Moreta (2019), Cadena, Pereira y Peréz (2019), Cuenca y González (2019).

De acuerdo a la sistematización expuesta en la tabla 6, se destaca que las empresas vinculadas a los principales productos de exportación, esto es banano, camarón y cacao, han implementado procesos para obtener certificaciones que respalden su gestión y calidad. En este sentido, las prácticas organizacionales analizadas se asocian a la innovación abierta, en virtud de que las certificaciones, de forma general, se alinean a protocolos orientados por asesores externos, normativas sanitarias de organismos internacionales y buenas prácticas que sirven de referencia. De manera que la innovación abierta se produce a partir de la transferencia de conocimientos que proviene principalmente de agentes externos.

En el caso del cacao, se identifica la innovación abierta en procesos de manufactura, por medio de distintos actores externos con el fin de mejorar su cadena de valor. Así, se destaca la iniciativa de la empresa Pacari al innovar sus procesos de producción con el apoyo agricultores de la provincia de Esmeraldas. Esta empresa también ha innovado en la creación de nuevos productos a partir de alianzas, es el caso de "Panettone Moderna Pacari", concebido en colaboración con la empresa panificadora "Moderna", (Revista Gestión, 2018).

Por otro lado, al contrastar los resultados con la literatura revisada se puede observar que las acciones implementadas por los sectores en estudio, también se asocian al tipo de innovación incremental, la cual se manifiesta como el mejoramiento paulatino y progresivo a partir de funcionalidades existentes en la organización (Sen y Ghandforoush, 2011; Fores y Camision, 2016) permitiendo reducción de costos y eficiencia en procesos.

Finalmente, de acuerdo a los hallazgos de Cadena et al. (2019), en un estudio aplicado al sector de alimentos y bebidas en la ciudad de Quito, se verifica que el 64\% de las empresas aplicaron la innovación radical en productos, lo que les permitió ampliar la oferta en el mercado. Del mismo estudio se desprende que la aplicación de innovación incremental en procesos representa el 56\% de las innovaciones, las estrategias de comercialización el 44,9\% y las mejoras 
en la estructura organizacional corresponden al $31 \%$ del total de las innovaciones identificadas. Este análisis se contrasta con la encuesta elaborada por el INEC y Senecyt (2015) en la cual se señala que el $60 \%$ de las empresas ecuatorianas implementaron un proceso significativamente mejorado.

\section{Conclusiones}

El propósito del artículo ha sido presentar un estudio descriptivo sobre los diferentes tipos de innovación que están aplicando las empresas exportadoras como estrategia para generar competitividad.

Tras el análisis empleado a la revisión de la literatura y a una muestra total de veinte publicaciones asociadas con nuestro objeto de estudio, se ha logrado identificar los tipos de innovación de mayor aplicación por parte de las empresas que operan en mercados internacionales, caracterizados por cambios continuos.

Entre los principales hallazgos constatamos que indiferentemente del tipo de innovación que apliquen las empresas, ya sea según su aplicación (producto, proceso, organización) o según su impacto (incremental o radical), estas se orientan bajo los paradigmas de la innovación cerrada y de la innovación abierta, así también con una combinación de ambas.

Tras el dinamismo de los mercados, la tendencia actual destaca la aplicación de la innovación abierta, en donde las empresas de diferentes sectores productivos utilizan como fuente de innovación las estrategias de alianzas con agentes externos, clientes, proveedores, competencia, estado y universidades (Rodríguez et al., 2011; Inauen y Schenker, 2012; Bernal y Frost, 2015; Tarapuez et al., 2016; García et al, 2016). Sin embargo, se observa que la innovación cerrada sigue siendo aplicada en ciertos sectores como en el caso de las firmas de alta tecnología (Inauen y Schenker, 2012).

Asociando la innovación abierta a los hallazgos en Ecuador, según la Encuesta Nacional de Actividades de Ciencia, Tecnología e Innovación (Inec y Senecyt, 2015), las cinco principales fuentes de información externas que las empresas usaron para innovar fueron: clientes y consumidores, internet, proveedores y competidores. Por lo que se constata la aplicación de innovación abierta como estrategia de competitividad.

Desde una perspectiva general, en el caso de Ecuador, se identifica que las empresas que aplican la innovación abierta utilizan prácticas organizacionales acorde a las exigencias del mercado, como es el caso de las certificaciones que requieren referentes externos de buenas prácticas, cooperación y transferencia de información. En este sentido, dado el nivel de rivalidad de los mercados en los que compiten los productos de exportación ecuatorianos, la innovación abierta se recomienda como una estrategia para defender la competitividad de las empresas.

Así también, de acuerdo al tipo de innovación según su impacto, la innovación incremental se destaca por su flexibilidad y efectividad, especialmente en procesos y estructura organizacional, contribuyendo a la competitividad a partir de la eficiencia en procesos y optimización de recursos. Un claro ejemplo está en el estudio al sector carrocero ecuatoriano 
(Bonilla et al., 2018), en donde la innovacion tecnológica redujo costos en procesos, provocando un incremento redituable en las empresas del sector.

Este ejemplo evidencia que la innovación en procesos con el uso de la tecnología permite optimizar y lograr procesos productivos mucho más eficientes, repercutiendo directamente en la rentabilidad de las empresas a corto y mediano plazo.

Finalmente, de acuerdo al enfoque cualitativo adoptado en esta investigación, a partir del análisis descriptivo basado en fuentes secundarias, se reconoce la limitante de no poder generalizar los resultados de los tipos de innovación identificados al contexto empresarial. No obstante, consideramos que este trabajo contribuye a enfatizar la importancia de fortalecer las capacidades de las empresas para adaptarse a los cambios mediante los diferentes tipos de innovación según sus necesidades. Mejorar los procesos de innovación a través de la gestión del conocimiento y la cooperación con agentes externos, robustecerá en las empresas su capacidad para definir estrategias que le permitan responder con un ritmo ajustado a la velocidad de las transformaciones de los mercados.

Por consiguiente, como futura línea de investigación se sugiere abordar el estudio de la innovación con un enfoque cuantitativo que permita medir el grado de influencia de las variables que facilitan la adopción de la innovación, así también, medir el impacto de los tipos de innovación en los resultados empresariales.

\section{Bibliografía}

Álvarez, E., \& Bernal, C. (2017). Modelo de Innovación Abierta: Énfasis en el Potencial Humano. Información Tecnológica, 28(1), 65-76. doi:10.4067/S0718-07642017000100007

Alvarez-Aros, E., \& Álvarez, M. (2018). Estrategias y prácticas de la innovación abierta en el rendimiento empresarial: Una revisión y análisis bibliométrico. Investigación Administrativa, $\quad 47(121), \quad 65-92 . \quad$ Obtenido de https://www.redalyc.org/jatsRepo/4560/456054552005/456054552005.pdf

Andrews, K. R. (1971). The Concept of Corporate Strategy. Homewood, Illinois: Dow JonesIrwin, Inc.

Arraut, L. (2008). La innovación de tipo organizacional en las empresas manufactureras de cartagena de indias. Semestre Económico, 11(22), 185-203. Obtenido de http://www.scielo.org.co/pdf/seec/v11n22/v11n22a9.pdf

Astudillo, S., \& Briozzo, A. (2016). Innovación en las mipymes manufactureras de Ecuador y Argentina. Semestre Económico, 19(40), 117-144. doi:10.22395/seec.v19n40a5

Badir, Y., Frank, B., \& Bogers, M. (09 de August de 2019). Employee-level open innovation in emerging markets: linking internal, external, and managerial resources. Journal of the Academy of Marketing Science, 1-23. doi:https://doi.org/10.1007/s11747-019-00674-6

Baldwin, C., \& Von Hippel, E. (2009). Modeling a Paradigm Shift: From Producer Innovation to User and Open Collaborative Innovation. Harvard Business School Finance Working Paper No. 10-038, 1-37. doi:https://dx.doi.org/10.2139/ssrn.1502864

Bernal, C., \& Blanco, C. (2017). Innovación por Diseño y su relación con las variables del entorno en una muestra de empresas en Bogotá-Colombia. Información Tecnológica, 28(4), 145156. doi:10.4067/S0718-07642017000400017 
Bernal, C., \& Frost, S. (2015). Innovación abierta en empresas colombianas: reto a superar. Revista $\begin{array}{llll}\text { Venezolana } & \text { Gerencia, 20 } & \text { 20(70), }\end{array}$ doi:http://dx.doi.org/10.31876/revista.v20i70.19996

Bonilla, D., Oña, B., \& López, H. (2018). Medición de innovación tecnológica como eje central del crecimiento empresarial familiar del sector carrocero de la provincia de Tungurahua. Revista Lasallista de investigación, 15(2), 271-285. doi:DOI: 10.22507/rli.v15n2a21

Cadena, J., Pereira, N., \& Peréz, Z. (2019). La innovación y su incidencia en el crecimiento y desarrollo de las empresas del sector alimentos y bebidas del Distrito Metropolitano de Quito (Ecuador) durante el 2017. Espacios, 40(22), 17. Obtenido de http://www.revistaespacios.com/a19v40n22/a19v40n22p17.pdf

Cano, M., \& Sánchez, G. (2014). El rol del capital intelectual en la innovacion de las empresas (Artículo de revisión). European Scientific Journal, 10(28), 348-366. Obtenido de http://eujournal.org/index.php/esj/article/viewFile/4405/4202

Cardinal, L., Alessandri, T., \& Turner, S. (2001). Knowledge codifiability, resources, and sciencebased innovation. Journal of Knowledge Management, 5(2), 195-204. doi:doi:10.1108/13673270110393266

Chandler, A. (1962). Strategy and Structure. Chapters in the History of the Industrial Enterprise. Massachusetts Institute of Technology.

Chesbrough, H. W. (2003). Open Innovation: The New Imperative for Creating and Profiting from Technology. Boston, Massachusetts: Harvard Business School Press.

Chesbrough, H. W. (2003). The logic of Open Innovation: Managing Intellectual Property. Carlifornia Management Review, 33-58. doi:https://doi.org/10.1177/000812560304500301

Chesbrough, H., \& Crowther, A. (2006). Beyond high tech: early adopters of open innovation in other industries. $R \& D$ Management, 36(3), 229-236. doi:10.1111/j.14679310.2006.00428.x

Chirinos, C., \& Rosado, J. (2016). Estrategia de diferenciación: el caso de las empresas industriales. Ingeniería Industrial(34), 165-174. Obtenido de https://www.redalyc.org/pdf/3374/337450992008.pdf

Cuenca-Macas, J., \& González-Illescas, M. (2019). Gestión del capital intelectual e innovación. Percepción de los exportadores de camaroón. Revista de Ciencia UNEMI, 12(30), 160-172. doi:http://dx.doi.org/10.29076/issn.2528-7737vol12iss30.2019pp160-172p

Da Silva, D., Vieira, R., Vieira, A., \& De Santiago, M. (2016). Optimización del proceso de innovación para proyectos internos en las empresas. Información Tecnológica, 27(3), 119130. doi:http://dx.doi.org/10.4067/S0718-07642016000300011

Damanpour, F. (1991). Organizational Innovation: A Meta-Analysis of Effects of Determinants and Moderators. The Academy of Management Journal, 34(3), 555-590. doi:https://doi.org/10.2307/256406

Dewar, R., \& Dutton, J. (1986). The Adoption of Radical and Incremental Innovations: An Empirical Analysis. Management Science, 32(11), 1422-1433. doi:http://dx.doi.org/10.1287/mnsc.32.11.1422

Ekos. (05 de Septiembre de 2012). Innovación tecnológica al servicio del sector bananero. Obtenido de Ekos Negocios: https://www.ekosnegocios.com/articulo/innovaciontecnologica-al-servicio-del-sector-bananero 
Ekos. (27 de Marzo de 2019). Innovación, la clave del crecimiento camaronero. Obtenido de Ekos Negocios: https://www.ekosnegocios.com/articulo/innovacion-la-clave-del-crecimientocamaronero

Enkel, E., Gassmann, O., \& Chesbrough, H. (2009). Open R\&D and open innovation: exploring the phenomenon. $\quad R \& D \quad$ Management, 39(4), 311-316. doi:http://dx.doi.org/10.1111/j.1467-9310.2009.00570.x

Escobar, P. (26 de Febrero de 2016). El chocolate nacional gana mercado. Obtenido de Revista Vistazo: https://issuu.com/vistazo.com/docs/aromas_sabores_web

Ettlie, J., Bridges, W., \& O'Keefe, R. (1984). Organization Strategy and Structural Differences for Radical Versus Incremental Innovation. Management Science, 30(6), 682-695. doi:https://doi.org/10.1287/mnsc.30.6.682

Forés, B., \& Camisón, C. (2016). ¿Does incremental and radical innovation performance depend on different types of knowledge accumulation capabilities and organizational size? Journal of business research, 69(2), 831-848. doi:https://doi.org/10.1016/j.jbusres.2015.07.006

Foronda, C. (2018). Características y efectos de la innovación en empresas de Bolivia: Una aplicación del modelo CDM. Investigación \& Desarrollo, 18(2), 57-72. doi:10.23881/idupbo.018.2-4e

Gálvez, E., \& García, D. (2012). Impacto de la innovación sobre el rendimiento de la mipyme: Un estudio empírico en Colombia. Estudios Gerenciales, 28(122), 11-27. doi:https://doi.org/10.1016/S0123-5923(12)70191-2

García-Pérez de Lema, D., Gálvez-Albarracín, E. J., \& Maldonado Guzmán, G. (2016). Efecto de la innovación en el crecimiento y el desempeño de las Mipymes de la Alianza del Pacífico. Un estudio empírico. Estudios Gerenciales, 32(141), 326-335. doi:https://doi.org/10.1016/j.estger.2016.07.003

Gómez, M., \& Etxebarria, G. (2012). Introducción: Variedad de temas y de tratamientos sobre la Innovación. Cuadernos de Gestión, 12(3), 15-26. doi:10.5295/cdg.120329mg

Greco, M., Grimaldi, M., \& Cricelli, L. (2016). An analysis of the open innovation effect on firm performance. European Management Journal, 34(5), 501-516. doi:https://doi.org/10.1016/j.emj.2016.02.008

Guisado González, M., Vila Alonso, M., \& Guisado Tato, M. (2016). Innovación, capacidad productiva, formación en el puesto de trabajo y productividad. Cuadernos de Gestión, 16(2), 77-92. doi:10.5295/cdg.140513mg

Gupta, A., \& Govindarajan, V. (2000). Knowledge Management's Social Dimension: Lessons From Nucor Steel. Sloan Management Review, 1.

Harkema, S. (2003). A complex adaptive perspective on learning within innovation projects. The Learning Organization, 10(6). doi:http://dx.doi.org/10.1108/09696470310497177

Inauen, M., \& Schenker-Wicki, A. (01 de April de 2012). Fostering radical innovations with open innovation. European Journal of Innovation, 15(2), 212-231. doi:https://doi.org/10.1108/14601061211220986

Inec y Senecyt. (2015). Encuesta Nacional de Actividades de Ciencia, Tecnología e Innovación 2012-2014. Recuperado el 6 de 12 de 2019, de Ecuadorencifras: https://www.ecuadorencifras.gob.ec/encuesta-nacional-de-actividades-de-cienciatecnologia-e-innovacion-acti/

Jamett, I., Alvarado, L., \& Maturana, S. (2017). Análisis al estado del arte de la innovación abierta: Implicaciones prácticas en la ingeniería. Revista Ingeniería de Construcción, 32(2), 73-84. doi:http://dx.doi.org/10.4067/S0718-50732017000200006 
Jordán, J. C. (2011). La innovación: una revisión teórica desde la perspectiva de marketing. Perspectivas(27), 47-71. Obtenido de https://www.redalyc.org/pdf/4259/425941231004.pdf

Kang, S., \& Hwang, J. (2019). An Investigation into the Performance of an Ambidextrously Balanced Innovator and Its Relatedness to Open Innovation. Journal of Open Innovation: Technology, Market, and Complexity, doi:https://doi.org/10.3390/joitmc5020023

Kanter, R. (1984). Innovation: Our Only Hope for Times Ahead? MIT Sloan Management Review, 25(4), 51-55.

Lakhani, K. R., Lifshiz Assaf, H., \& Tushman, M. L. (2013). Open innovation and organizational boundaries: Task decomposition, knowledge distribution and the locus of innovation. En A. Grandori (Ed.), Handbook of Economic Organization: Integrating Economic and Organization Theory (págs. 355-382). Northampton, MA: Edward Elgar Publishing. doi:10.4337/9781782548225.00030

Liao, S.-H., Fei, W.-C., \& Chen, C.-C. (2007). Knowledge Sharing, Knowledge Absorptive Capacity, and Innovation Capability: An Empirical Study in Taiwan Knowledge Intensive Industries. Journal of Information Science, 33(3), 340-359. doi:https://doi.org/10.1177\%2F0165551506070739

Líderes. (16 de Mayo de 2017). La innovación llega a los sistemas de cosecha de cacao. Obtenido de Revista Líderes: https://www.revistalideres.ec/lideres/innovacion-sistemas-cacaoimportacion-tecnologia.html

López-Sánchez, J. I., Minguela-Rata, B., Rodríguez-Duarte, A., \& Sandulli, F. D. (2006). Innovaciones incrementales e innovaciones radicales: un estudio de las características intrínsecas de los equipos para el desarrollo de nuevos productos. Cuadernos de Estudios Empresariales, $\quad 16, \quad 33-53 . \quad$ Obtenido de https://revistas.ucm.es/index.php/CESE/article/view/CESE0606110033A/9463

March, J., \& Simon, H. (1958). Organizations. New York: John Wiley \& Sons.

Montoya Suárez, O. (2004). Schumpeter, innovación y determinismo tecnológico. Scientia et Technica, 2(25), 209-213. doi:http://dx.doi.org/10.22517/23447214.7255

Moreta, M. (28 de Mayo de 2019). La innovación llegó al calcetín. Obtenido de Líderes: https://www.revistalideres.ec/lideres/innovacion-calcetin-industria-medicinaproductos.html

Nelson, R., \& Winter, S. (1982). An evolutionary theory of economic change. Harvard University Press.

Nonaka, I., \& Takeuchi, H. (1995). The Knowledge-Creating Company. New York: Oxford University Press.

Norman, D., \& Verganti, R. (2014). Incremental and Radical Innovation: Design Research vs. Technology and Meaning Change. Design Issues, 30(1), 78-96. doi:doi:10.1162/DESI_a_00250

OCDE y Eurostat. (2006). Oslo Manual 3rd Edition: Guidelines for Collecting and Interpreting Innovation Data (3rd Edition ed.). Obtenido de http://www.itq.edu.mx/convocatorias/manualdeoslo.pdf

OECD y Eurostat. (2018). Oslo Manual 2018: Guidelines for Collecting, Reporting and Using Data on Innovation (4th Edition ed.). Paris. doi:https://doi.org/10.1787/9789264304604en

Ohmae, K. (1989). La mente del estratega (Primera ed.). México: McGraw Hill. 
Orlikowski, W. (1991). Radical and incremental innovations in systems development: an empirical investigation of case tools. Center for Information Systems Research(221), 1-27.

Paspuel, W. (25 de Octubre de 2015). La innovación impulsa al sector camaronero. (W. Paspuel, Ed.) Obtenido de Líderes: https://www.revistalideres.ec/lideres/produccion-camaroninnovacion-exportaciones.html

Pavón, A., Hernández, A., Suárez, J., Jiménez, B., \& Sánchez, V. (2015). Análisis de los modelos de Innovación Abierta. Ventajas de su aplicación. Revista Avanzada Científica, 18(3), 3244. Obtenido de https://dialnet.unirioja.es/servlet/articulo?codigo=5265921

Polo, J., Ramos, J., Arrieta, A., \& Ramirez, N. (2018). Impacto de la innovación sobre la conducta exportadora en el sector de alimentos y bebidas de Colombia. Revista de Análisis Económico, 33(1), 89-210. doi:http://dx.doi.org/10.4067/S0718-88702018000100089

Porter, M. (1980). Competitive Strategy: Techniques for Analyzing Industries and Competitors (Primera ed.). New York: Free Press.

Porter, M. (1985). Competitive Advantage. New York: The Free Press.

Porter, M. (Marzo-Abril de 1990). The Competitive Advantage of Nations. Harvard Business Review, 73-91.

Rajapathirana, R., \& Hui, Y. (2018). Relationship between innovation capability, innovation type, and firm performance. Journal of Innovation \& Knowledge, 3(1), 44-55. doi:https://doi.org/10.1016/j.jik.2017.06.002

Revista Gestión. (20 de Noviembre de 2018). Moderna y Pacari anuncian el inicio de su alianza de innovación de productos. Obtenido de Revista Gestión: https://revistagestion.ec/empresas/moderna-y-pacari-anuncian-el-inicio-de-su-alianza-deinnovacion-de-productos

Rodríguez, C., Terán, A., \& Bucci, N. (2011). La innovación abierta como elemento de análisis en las pequeñas y medianas industrias. Caso sector metalmecánico. Revista de Administração e Inovação, 8(2), 5-28. doi:10.5773/rai.v8i2.595

San-Martín, N., \& Rodríguez-Castellanos, A. (2012). Un marco conceptual para los procesos de innovación abierta: integración, difusión y cooperación en el conocimiento. Telos, 14(1), 83-101. Obtenido de https://www.redalyc.org/articulo.oa?id=99322258006

Schmookler, J. (1966). Invention and economic growth. Harvard University Press.

Schumpeter, J. A. (1934). The Theory of Economic Development. Cambridge, Massachusetts: Harvard University.

Sen, T., \& Ghandforoush, P. (2011). Radical and Incremental Innovation Preferences in Information Technology: An Empirical Study in an Emerging Economy. Journal of Management Technology \& Innovation, 6(4), 33-44. doi:https://doi.org/10.4067/S071827242011000400003

Sherman, G. (1981). Technology Transfer Innovation and International Competitiveness. John Wiley \& Sons Inc.

Shih, T.-Y. (04 de June de 2018). Determinants of enterprises radical innovation and performance: Insights into strategic orientation of cultural and creative enterprises. Sustainability, 10, 22. doi:10.3390/su10061871

Tarapuez, E., Guzmán, B., \& Parra, R. (2016). Estrategia e innovación en las Mimpymes colombianas ganadoras del premio Innova 2010-2013. Estudios Gerenciales, 32(139), 170180. doi:https://doi.org/10.1016/j.estger.2016.01.002 
Teece, D. (1986). Profiting from technological innovation: Implications for integration, collaboration, licensing and public policy. Research Policy, 15(6), 285-305. doi:https://doi.org/10.1016/0048-7333(86)90027-2

Tudor, A. T., Zaharie, M., \& Osoian, C. (2014). Innovation development needs in manufacturing companies. Procedia Technology, 12, 505-510. doi:10.1016/j.protcy.2013.12.522

Velásquez, S., Pino, A., Restrepo, E., \& Viana, N. (29 de Noviembre de 2018). Innovación en empresas: estado del arte considerando tendencias para su implementación. Espacios, 39(48), 7. Obtenido de http://www.revistaespacios.com/a18v39n48/a18v39n48p07.pdf

Vistazo. (23 de Febrero de 2018). El sector textil hila firme. Obtenido de Revista Vistazo: https://issuu.com/vistazo.com/docs/enfoque__

Wang, C., \& Ahmed, P. (2004). The development and validation of the organisational innovativeness construct using confirmatory factor analysis. European Journal of Innovation Management, 7(4), 303-313. doi:https://doi.org/10.1108/14601060410565056 